\title{
TI.106.1
}

\section{Identity Provider Strategies for Common Campus Environments}

- PDF: IdentityProviderStrategiesforCommonCampusEnvironments-FINAL.pdf

- Text: IdentityProviderStrategiesforCommonCampusEnvironments-FINAL.txt

\section{More Information}

\begin{tabular}{|c|c|}
\hline Repository ID & TI.106.1 \\
\hline Persistent URL & http://doi.org/10.26869/TI.106.1 \\
\hline Title & Identity Provider Strategies for Common Campus Environments \\
\hline Authors & Janemarie Duh, David Walker <https://orcid.org/0000-0003-2540-0644>, Alternative Identity Providers Working Group \\
\hline Sponsor & InCommon TAC \\
\hline \multicolumn{2}{|l|}{ Review } \\
\hline Status & Legacy \\
\hline Publish Date & December, 2014 \\
\hline DOI & 10.26869/TI.106.1 \\
\hline \multicolumn{2}{|l|}{ Signature } \\
\hline Deprecated & No \\
\hline \multicolumn{2}{|l|}{ Future Review } \\
\hline \multicolumn{2}{|l|}{ Supersedes } \\
\hline Format & PDF, Text \\
\hline \multicolumn{2}{|l|}{ Related Docs } \\
\hline Development Location & https://spaces.at.internet2.edu/x/oQLkAg \\
\hline \multicolumn{2}{|l|}{ IP Framework } \\
\hline Subject Tags & federation \\
\hline Notes & \\
\hline
\end{tabular}

